NOTE TECHNIQUE

\title{
INFLUENCE DE LA TAILLE DES PARTICULES ALIMENTAIRES SUR LA CROISSANCE DU SAUMON ATLANTIQUE LORS DE LA PRISE D'ALIMENTATION
}

\author{
J.L. GAIGNON, J.C. ALEXANDRE, A. LE ROUX \\ Centre Océanologique de Bretagne - BP 337 - 29273 BREST CEDEX
}

\begin{abstract}
RÉSUMÉ
Les taux de croissance enregistrés sur des saumons atlantiques en période de première alimentation ont permis de montrer que la formule établie pour des parrs par WANKOWSKI : taille des particules à distribuer $=$ taille du saumon $\times 0,024$ est vérifiée pour des alevins de plus de $250 \mathrm{mg}$ pour différents aliments. Pour des alevins de taille inférieure à $250 \mathrm{mg}$, les résultats dépendent de l'aliment, une taille d'aliment de 400 $630 \mu$ permet en général des performances satisfaisantes. Pratiquement on détermine une correspondance entre taille d'aliment à distribuer et le poids de l'alevin pour la période d'alevinage.
\end{abstract}

\section{ABSTRACT}

For different food, the growth rates show that the food particule-size to fish length ratio established by WANKOWSKI for atlantic salmon parr is good for feeding fry atlantic salmon weighing $250 \mathrm{mg}$ or more. For fry-weight below $250 \mathrm{mg}$, the growth rate depends on the food, but generally a $400-600 \mu$ food particule-size gives good growth. Practically, we establish the different particule-size to feed atlantic salmon fry of different weight.

\section{INTRODUCTION}

Les taux de croissance enregistrés dans les élevages de saumon atlantique /Sa/mo salar) du COB durant la phase de première alimentation ont été jusqu'à maintenant particulièrement faibles (inférieurs à $1 \% /$ jour) durant les 10-20premiers jours (GAIGNON et PROUZET, 1982 b). Plusieurs causes peuvent expliquer ces résultats : conditions d'incubation - résorption peu favorables (GAIGNON et PROUZET, 1982 a), méthodes d'élevage peu performantes (paramètres d'élevage non optimisés) ou "problèmes alimentaires" au sens large. Dans ce document nous aborderons partiellement le dernier point qui peut recouvrir de nombreux aspects: la valeur nutritionnelle, l'appétence (qui peut être fonction de la composition chimique et/ou de caractéristiques physiques: structure, texture, couleur, densité par rapport à l'eau, calibre,...), la distribution (durée, fréquence, méthode,...).

La taille de l'aliment a déjà fait l'objet d'études (WANKOWSKI and THORPE, 1979) qui montrent que, durant toute la durée du cycle de production en eau douce, excepté pour les alevins, la taille de la particule ingérée est reliée à la taille du saumon atlantique par la relation:

$\frac{\text { Taille des particules }}{\text { Taille du saumon }}=\operatorname{PFR}(1)=0,022$ a 0,026

(1) Abréviation anglaise pour : "Food particule-size to fish length ratio". 
Pour la période du "démarrage" alimentaire la relation est beaucoup moins précise et la meilleure croissance en poids est obtenue avec un PFR de 0,011-0,090.

Les calibres que nous employons habituellement sont très différents de ceux qui permettent à WANKOWSKI et THORPE (1979) d'obtenir les meilleures performances. De plus leurs résultats ont été obtenus dans des conditions d'environnement, dans des structures et avec des aliments différents de ceux que nous utilisons. Afin de déterminer si le paramètre "taille de l'aliment" pouvait être en partie la cause des médiocres résultats passés, nous avons donc entrepris d'étudier l'influence de la taille des particules alimentaires, en relation avec la taille de l'alevin, sur les performances de croissance, lors de la période de première alimentation (période de 15 jours - 3 semaines) et ceci pour différentes formules alimentaires.

\section{I - MATÉRIEL et MÉthode}

\subsection{Moyens expérimentaux}

Les différentes expériences ont été effectuées sur des alevins issus de géniteurs capturés dans le ruisseau Saint-Jean, affluent de l'Elorn. Les œufs ont été incubés à la pisciculture du Quinquis puis transportés au Centre Océanologique de Bretagne où se sont déroulés les essais. Les structures expérimentales sont des bacs type "suédois " de $0,50 \times 0,50 \mathrm{~m}$, non couverts, éclairés en lumière artificielle durant la phase diurne.

Les aliments sont distribués automatiquement de façon continue et la ration journalière est d'environ $10 \%$ du poids total, taux identique pour chaque lot d'une même expérience. Les régimes utilisés sont un aliment commercial GSO(1), une formule spéciale SANDERS (2) et une formule COB (3), dénommés par la suite respectivement "J", "F", "R".

Les analyses proximales de ces aliments figurent en Annexe I. On note que:

- Les aliments " $R$ " et " $J$ " ont une teneur voisine en protéines, lipides et cendres, le " $J$ " étant légèrement moins riche en lipides (12-14\% au lieu de 14-15\%).

- L'aliment " $F$ " se différencie des deux autres par:

- une plus faible teneur en protéines (52-53\% au lieu de $60 \%$ )

- une teneur en lipides légèrement supérieure (16-17\% au lieu de $12-15 \%)$

- une quantité de matières minérales nettement plus faible $(7,5 \%$ au lieu de $12-14 \%)$.

Pour chaque aliment il n'y a pas de différence de composition en fonction du calibre. Les granulométries industrielles étant très hétérogènes (cf. Annexe II), chaque aliment est calibré sur tamis standard au laboratoire.

\subsection{Protocoles expérimentaux}

La première expérience devait nous permettre de déterminer la taille la plus adéquate sur la base des données de WANKOWSKI, et ce avec un seul aliment " J" couramment utilisé dans nos élevages (cf. tableau 1). Les calibres retenus sont donc 200$400,400-600,600-800,800-1000 \mu$ (soit un PFR $=0,01$ a 0,04 ). Les résultats obtenus n'ont pas été satisfaisants et nous ont contraints de modifier le protocole par la suite. Ainsi les protocoles expérimentaux diffèrent pour chaque expérience en fonction des résultats de l'essai précédent. Dans la seconde expérience, des calibres plus petits et deux aliments ont été utilisés; les densités d'élevage sont modifiées. La troisième expérience est mise en place pour confirmer les résultats obtenus dans les deux expériences précédentes. Les protocoles des trois séries expérimentales sont résumés dans le tableau 1. Les lots ne sont pas répliqués pour une expérience donnée.

\subsection{Recueil des données}

Pour chaque expérience, une répartition avec comptage des alevins est effectuée lors de la mise en charge. Par la suite les mortalités sont relevées quotidiennement.

Les poids moyens sont déterminés par la pesée individuelle, toutes les semaines, d'une quarantaine d'alevins de chaque bac a la balance de précision (précision de l'ordre du mg).

(1) Grande Semoulerie de l'Ouest - 16160 LE GOND PONTOUVRE

(2) SANDERS - 17, quai de I'Industrie - 92100 ATHIS MONS

(3) L'aliment est établi et fabriqué par le laboratoire de nutrition du CNEXO/COB. 


\begin{tabular}{|c|c|c|c|}
\hline & Expérience 1 & Expérience $z$ & Expérience 3 \\
\hline $\begin{array}{l}\text { Age el poids moyen } \\
\text { en début d'expérience }\end{array}$ & $\begin{array}{c}\text { fin résorption } \\
0.147 \mathrm{~g}\end{array}$ & $\begin{array}{c}\text { fin résorption } \\
0.127 \varepsilon\end{array}$ & $\begin{array}{l}\text { démarrú: cn } \\
\text { pisciculture } \\
\text { sur alinont } \\
\text { dutre que } \\
\text { ceux testés } \\
\text { (1) } 0,1758\end{array}$ \\
\hline $\begin{array}{c}\text { Température de l'eau } \\
\text { en }{ }^{\circ} \mathrm{C}\end{array}$ & 9,6 ̀े 13 & 12 à 13,5 & 12,5 ¿े 14,0 \\
\hline $\begin{array}{l}\text { Densité en nombre } \\
\text { alevins/m2 }\end{array}$ & 480 & 920 & 840 \\
\hline Aliment(s) Utilisé(s) & $\mathrm{J}$ & $J$ et $F$ & $J, F, R$ \\
\hline $\begin{array}{l}\text { Taille de l'aliment } \\
\text { utilisé en } \mu \\
\text { (tamis standard) }\end{array}$ & $\begin{array}{l}200-400 \\
400-630 \\
630-800 \\
800-1000\end{array}$ & $\begin{array}{r}<200 \\
200-400 \\
400-630\end{array}$ & $\begin{array}{l}200-400 \\
400-630 \\
630-800\end{array}$ \\
\hline
\end{tabular}

(1) : expérience effectuée trop tard derıs la salson et tous les alevins étaient "démarrés"

\section{Tableau 1: Évolution des protocoles mis en place.}

Les quantités d'aliment distribuées sont pesées quotidiennement.

\subsection{Traitement des données}

Pour chaque donnée, on fournit la moyenne et l'erreur standard. Une analyse de variance est effectuée pour chaque expérience; si une différence significative existe $(p=0,05)$, les données sont classées grâce au test a posteriori de Student Newman Keuls.

\section{II - RÉSULTATS}

\subsection{Expérience 1}

Quatre semaines après la première distribution alimentaire, les mortalités enregistrées sont identiques et négligeables ( 1 ou 2 alevins sur 119) sur tous les bacs.

D'autre part, on observe une absence totale de croissance (fig. 1) durant les deux premières semaines quel que soit le calibre utilisé. Par la suite, plus l'aliment est gros et plus le démarrage est tardif. Les meilleurs résultats sont donc obtenus avec le plus petit calibre $(200-400 \mu)$; la croissance moyenne obtenue avec ce calibre est de $1,71 \% / \mathrm{j}$.

Alors que la croissance n'a pas démarré, la structure de la population du lot alimenté avec le calibre 800-1000 $\mu$ montre le 18 mars une fréquence élevée d'alevins de petite taille (figure 2). Le $\mathbf{2 5}$ mars, le poids moyen a augmenté (différence significative) et l'on observe une fréquence élevée d'alevins de grosse taille (figure 2), c'est-à-dire que les plus petits alevins ont dans leur ensemble grossi. Ceci montre que ce calibre, bien que relativement gros, est ingéré par tous les alevins. 


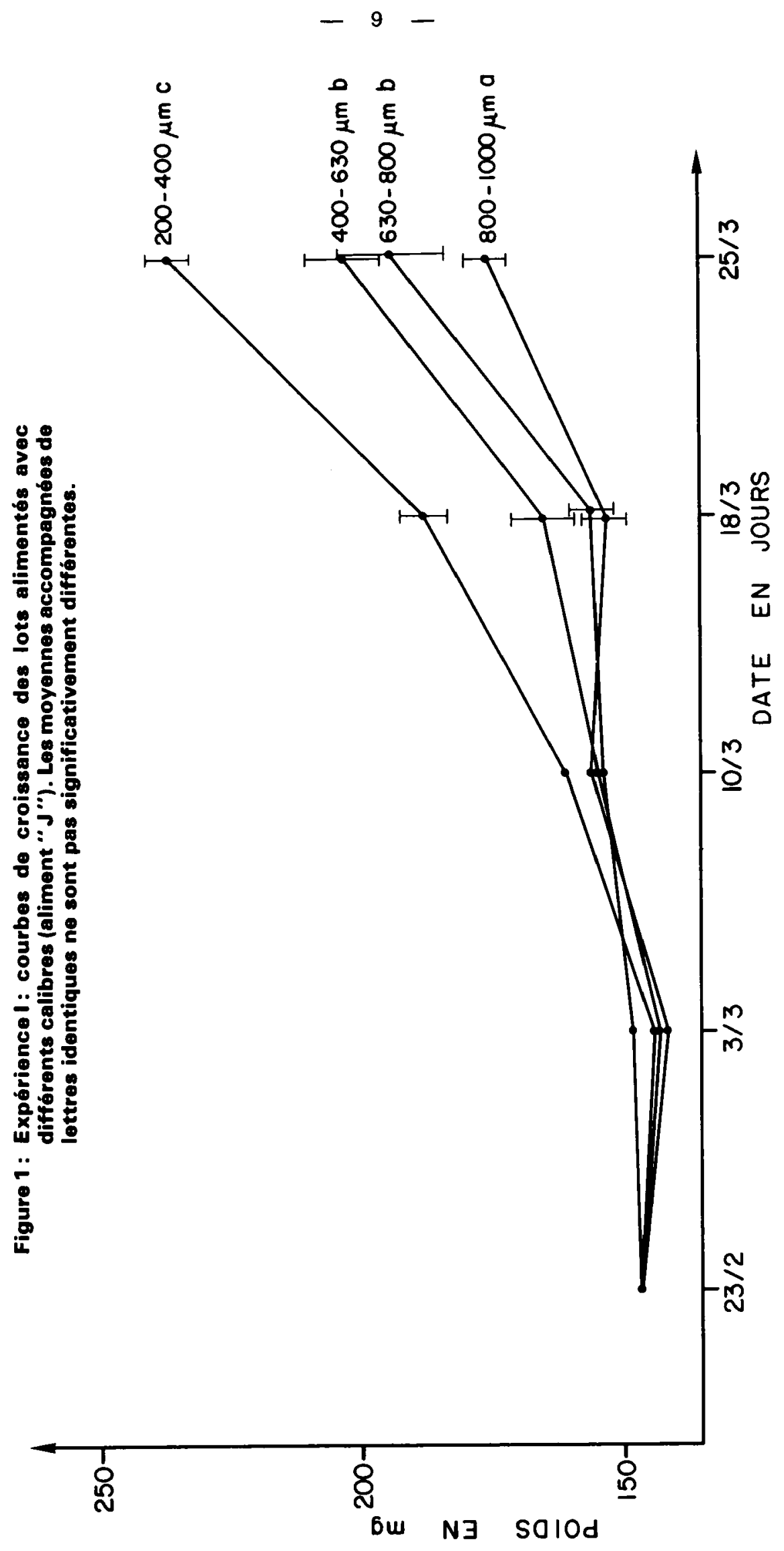




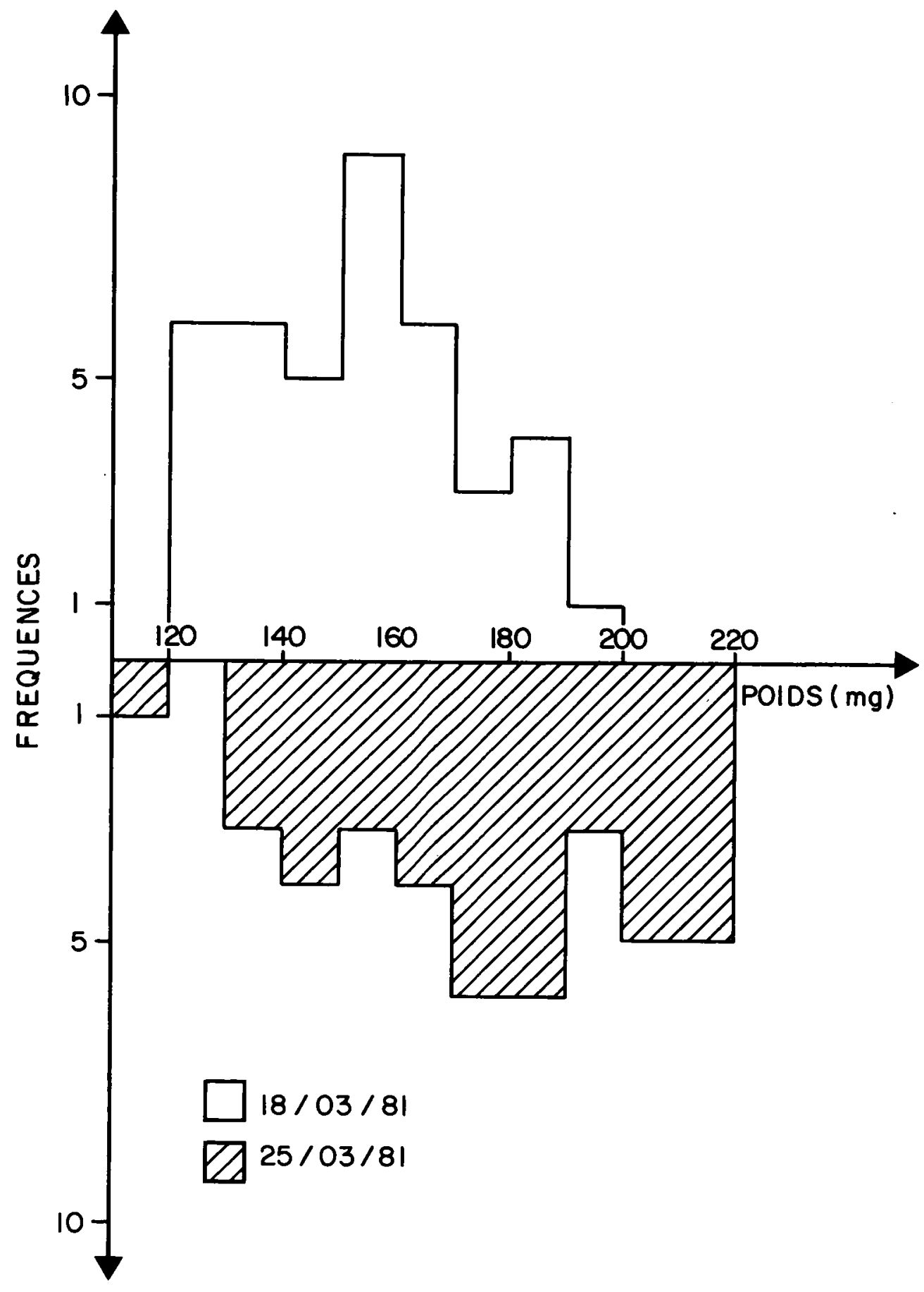

Figure 2 : Expérience I : Évolution des histogrammes de fréquences des lots alimentés avec le calibre $800-1000 \mu$.

\subsection{Expérience 2}

Les mortalités enregistrées, comprises entre 4,8 et $7 \%$ pour les différents calibres et aliments sur trois semaines sont relativement fortes mais non significativement différentes $\left(\chi^{2}=0,143\right)$ en fonction des lots. 


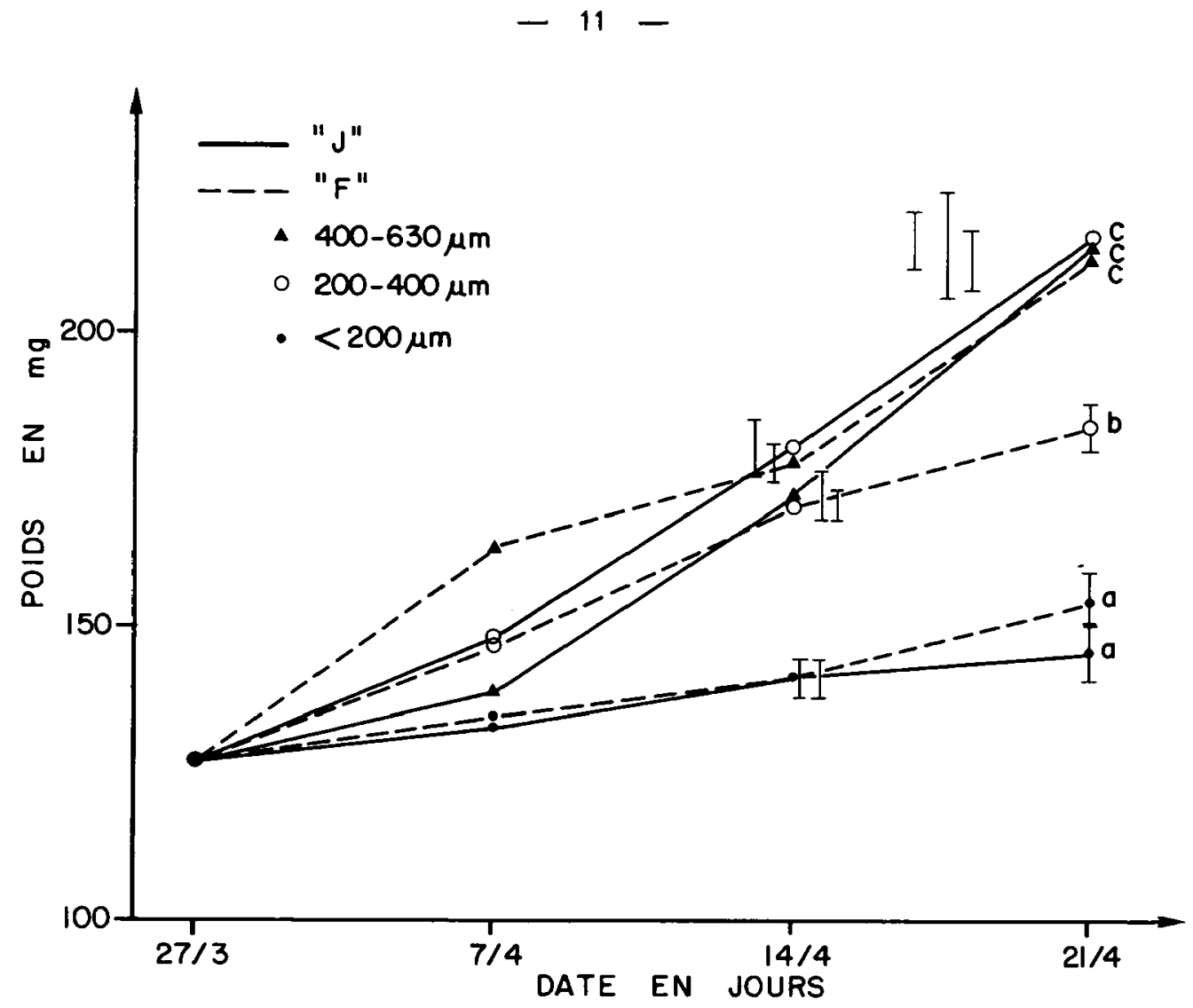

Figure 3 : Expérience II: courbes de croissance obtenue pour des lots recevant les aliments "J" et "F" de différents calibres. Les moyennes accompagnées de lettres identiques ne sont pas significativement diffórentes.

La figure 3 montre que pour les lots alimentés avec le calibre inférieur à $200 \mu$ on n'observe aucune croissance, par contre, dans les autres cas il y a un gain de poids dès le départ. Pour l'aliment " $J$ ", il n'y a pas de différence significative de poids moyen entre les lots alimentés avec les calibres 200-400 et 400-630 $\mu$; le calibre 200-400 $\mu$ permet donc d'obtenir des performances au moins identiques aux calibres les plus performants, ce qui confirme les résultats de l'expérience 1. Par contre, pour l'aliment " $F$ " il y a une différence significative entre ces deux calibres (sauf le 14/4 : problèm d'échantillonnage) et, contrairement à l'aliment " $J$ ", les meilleurs résultats sont obtenus avec le calibre $400-630 \mu$.

La comparaison d'aliments montre que pour le calibre $400-630 \mu$, l'aliment " $F$ " semble permettre un démarrage nettement plus rapide que celui observé avec l'aliment " $J$ ". La croissance obtenue est de 3,60\%/jour. Cependant, il n'y a plus de différence significative de poids moyen en fin d'expérience. Pour le calibre $200-400 \mu$, l'aliment " $J$ " permet les meilleures performances de croissance et la différence avec l'aliment " $F$ " s'accroît régulièrement.

\subsection{Expérience 3}

Les mortalités enregistrées, comprises entre 3,8 et $9,0 \%$ pour les différents calibres et aliments de cette expérience, sont relativement fortes mais non significativement différentes $\left(\chi^{2}=3,2\right)$.

Des différences de croissance sont enregistrées dès le début de l'essai. Le meilleur résultat est obtenu durant la première semaine sur le lot alimenté avec du " $\mathrm{J}$ " calibre $200-400 \mu$ (lot " $\mathrm{J} " / 200-400)(5,56 \% /$ jour) (cf. tableau 2), mais sur l'ensemble 
de l'expérience (cf. figure 4), le lot " $R$ "/630-800 (4,31\%/jour) a les meilleures performances. Globalement on remarquera (cf. figure 4) que, pour chaque aliment, la meilleure croissance est obtenue avec le calibre $630-800 \mu$.

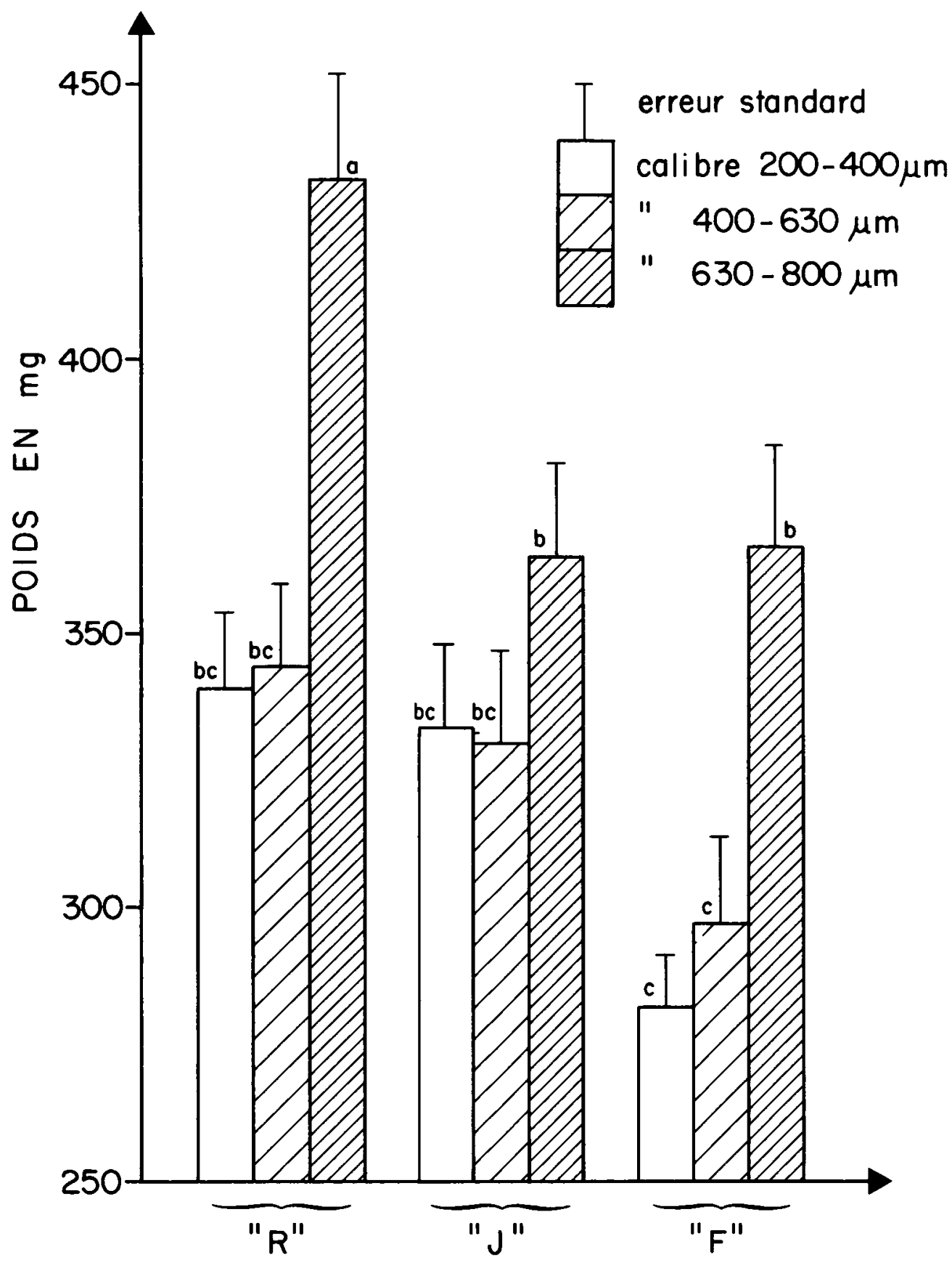

Figure 4 : Expérience III : poids moyen en fin d'expérience $(26 / 5)$ pour chaque lot en $f$ inction de l'aliment. Les moyennes accompagnées de lettres identiques ne sont pas significativement différentes. 
Tableau 2: Expérience III : Résultats détaillés des différents échantilonnages.

\begin{tabular}{|c|c|c|c|c|c|c|c|}
\hline Aliment & $\begin{array}{c}\text { Calibre } \\
\text { en } \mu\end{array}$ & $\begin{array}{c}\text { Echant } \\
\text { du } 1\end{array}$ & $\begin{array}{l}\text { lonnage } \\
5 / 82\end{array}$ & $\begin{array}{c}\text { Echant } \\
\text { de } 1\end{array}$ & $\begin{array}{l}\text { lonnage } \\
5 / 82\end{array}$ & $\begin{array}{l}\text { Echant } \\
\text { du } 2\end{array}$ & $\begin{array}{l}\text { lonnage } \\
5 / 82\end{array}$ \\
\hline \multirow{3}{*}{ "R" } & $200-400$ & $\begin{array}{l}240 \\
29,9\end{array}$ & $\begin{array}{l}4,48 \\
0.01\end{array}$ & $\begin{array}{r}335,1 \\
32,2\end{array}$ & $\begin{array}{l}4,77 \\
0,009\end{array}$ & $\begin{array}{r}346,1 \\
32,5\end{array}$ & $\begin{array}{l}0,46 \\
0,009\end{array}$ \\
\hline & $400-630$ & $\begin{array}{r}237,1 \\
29,8\end{array}$ & $\begin{array}{l}4,31 \\
0,017\end{array}$ & $\begin{array}{r}323,3 \\
31,9\end{array}$ & $\begin{array}{l}4,43 \\
0,016\end{array}$ & $\begin{array}{r}344,6 \\
32,4\end{array}$ & $\begin{array}{l}0,91 \\
0,015\end{array}$ \\
\hline & $630-800$ & $\begin{array}{r}247,7 \\
30,1\end{array}$ & $\begin{array}{l}4.93 \\
0,023\end{array}$ & $\begin{array}{r}361.1 \\
32,8\end{array}$ & $\begin{array}{l}5.38 \\
0.021\end{array}$ & $\begin{array}{r}433,2 \\
34,5\end{array}$ & $\begin{array}{l}2,60 \\
0,02\end{array}$ \\
\hline \multirow{3}{*}{$m n$} & $200-400$ & $\begin{array}{r}258,8 \\
30,4\end{array}$ & $\begin{array}{l}5,56 \\
0,01\end{array}$ & $\begin{array}{r}300,0 \\
31,3\end{array}$ & $\begin{array}{l}2.11 \\
0.01\end{array}$ & $\begin{array}{r}333,1 \\
32,1\end{array}$ & $\begin{array}{l}1,49 \\
0,011\end{array}$ \\
\hline & $400-630$ & $\begin{array}{r}226,6 \\
29,6\end{array}$ & $\begin{array}{l}3,66 \\
0,01\end{array}$ & $\begin{array}{c}245,4 \\
-\end{array}$ & - & $\begin{array}{c}329,5 \\
-\end{array}$ & $\begin{array}{l}- \\
-\end{array}$ \\
\hline & $630-800$ & $\begin{array}{r}230,8 \\
29,7\end{array}$ & $\begin{array}{l}3,92 \\
0,017\end{array}$ & $\begin{array}{r}299,3 \\
31,3\end{array}$ & $\begin{array}{l}3,71 \\
0,016\end{array}$ & $\begin{array}{r}364,5 \\
32,9\end{array}$ & $\begin{array}{l}2,81 \\
0,015\end{array}$ \\
\hline \multirow{3}{*}{${ }^{n} F^{n}$} & $200-400$ & $\begin{array}{r}218,9 \\
29,4\end{array}$ & $\begin{array}{l}3,16 \\
0,01\end{array}$ & $\begin{array}{r}260.7 \\
30,4\end{array}$ & $\begin{array}{l}2.50 \\
0.01\end{array}$ & $\begin{array}{r}281,9 \\
30,9\end{array}$ & $\begin{array}{l}1,12 \\
0,01\end{array}$ \\
\hline & $400-630$ & $\begin{array}{r}210,9 \\
29,2\end{array}$ & $\begin{array}{l}2,63 \\
0,017\end{array}$ & $\begin{array}{r}271,8 \\
30,7\end{array}$ & $\begin{array}{l}3,62 \\
0,016\end{array}$ & $\begin{array}{r}296,8 \\
31,3\end{array}$ & $\begin{array}{l}1,26 \\
0,016\end{array}$ \\
\hline & $630-800$ & $\begin{array}{r}204,5 \\
29,1\end{array}$ & $\begin{array}{l}2,19 \\
0,024\end{array}$ & $\begin{array}{r}308,0 \\
31,5\end{array}$ & $\begin{array}{l}5,85 \\
0,022\end{array}$ & $\begin{array}{r}366,0 \\
32,9\end{array}$ & $\begin{array}{l}2,46 \\
0,021\end{array}$ \\
\hline
\end{tabular}

Poids moyen initial le 5/5/82 : $175,4 \mathrm{mg}$

Pour chaque lot et à chaque date, on donne :

- le poids moyen en $\mathrm{mg}$ ( $P \mathrm{mg})$

- le taux de croissance en $\% / j$

- la longueur moyenne ( $L \mathrm{~mm}$ )- en mm calculée d'après la relation

$P \mathrm{mg}=42 \mathrm{~L} \mathrm{~mm}$ - 1017 (utilisable jusqu'à $45 \mathrm{~mm}$ )

- PFR calculé d'après la relation : taille moyenne des particules

taille du saumon

$$
\begin{aligned}
& \text { P mg Croissance } \\
& L \mathrm{~mm} \quad \text { PFR }
\end{aligned}
$$


Pour les aliments " $R$ " et " $F$ " on notera que pour un poids d'alevin déterminé et supérieur à $250 \mathrm{mg}$ (cf. tableau 2 et figure 5), les meilleurs taux de croissance sont obtenus sur les lots ayant un PFR compris entre 0,020 et 0,023 . Sur la durée de l'expérience, les gains de poids de ces lots par rapport aux lots ayant un PFR inférieur ne font que s'accroître.
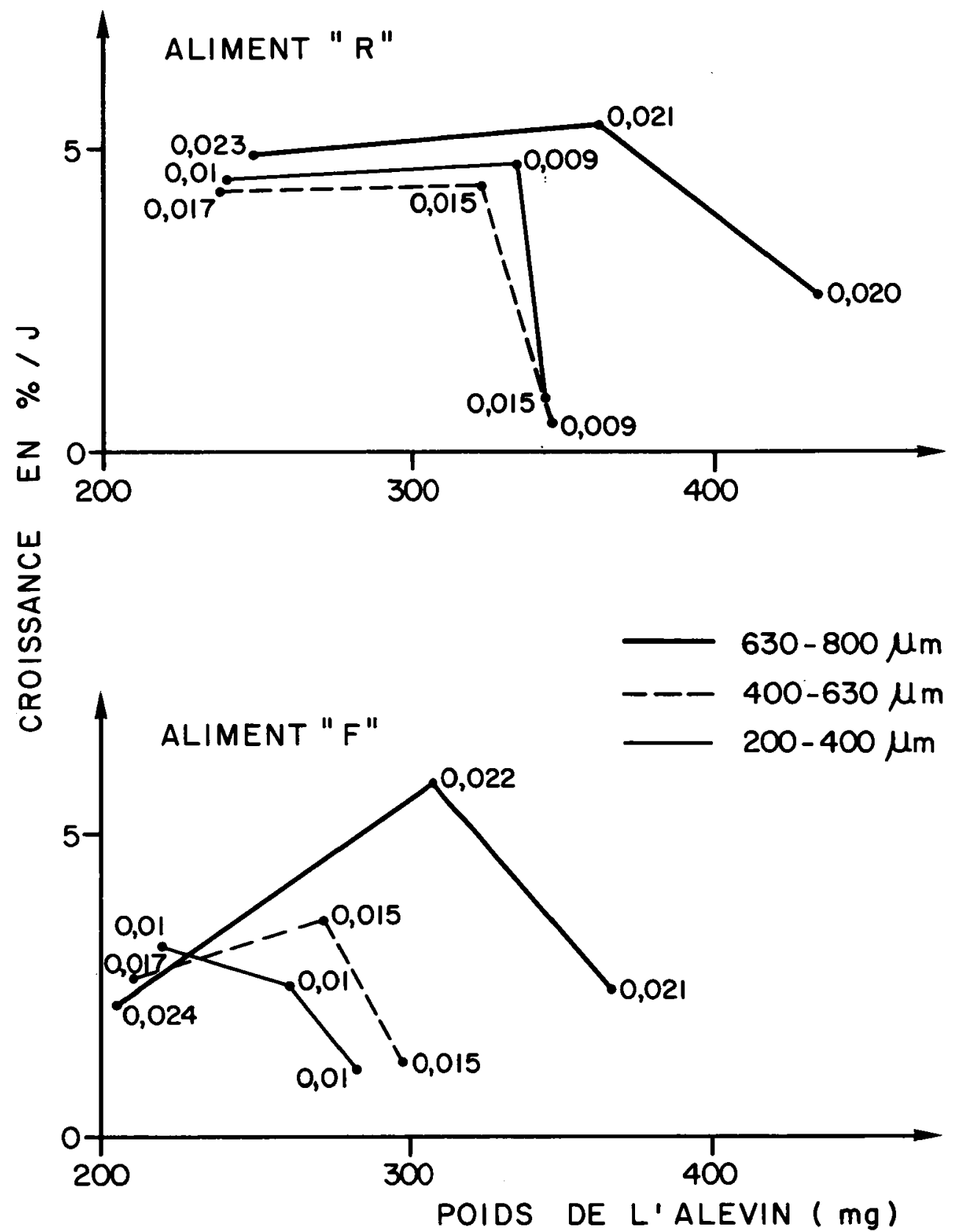

Figure 5 : Expérience III : Évolution du taux de croissance moyenne en poids des différents lots en fonction du poids moyen des alevins des lots et de la taille des particules pour deux aliments (" $R$ " ot " $F$ "). Pour chaque point d'échantillonnage on donne le PFR. 


\section{III - DIscussion}

1. Comme les conditions expérimentales diffèrent d'une expérience à l'autre et que les températures de l'eau augmentent, il n'est pas possible de comparer les données brutes entre elles; seule une comparaison relative peut être tentée.

2. Lors du pré-traitement des données du premier essai, il est apparu que les densités d'alevins utilisées pouvaient avoir une incidence sur les performances de croissance. Aussi, par la suite, elles ont été augmentées (de 480 à environ 900 alevins $/ \mathrm{m}^{2}$ ), tous les autres paramètres d'élevage restant identiques. Les résultats s'étant améliorés, on supposera que l'absence de croissance enregistrée durant le premier essai peut être due à ce facteur. Or, les densités utilisées sont en moyenne inférieures à celles pratiquées en élevage (REFSTIE et KITTELSEN, 1976; GAIGNON et PROUZT, 1981), parfois identiques (CANYURT, 1976) mais supérieures à celles utilisées pour les expériences en aquarium (par exemple FENDERSON et CARPENTIER, 1971). On peut alors penser qu'un facteur lié à la densité ait pu avoir une influence. Ce pourrait être la densité des particules alimentaires disponibles. En effet, les rations d'aliment distribuées sont établies en pourcentage du poids vif, c'est-à-dire que pour une charge identique, plus les particules sont grosses et moins il $y$ en a par unité de volume d'élevage (dans un rapport 1/27 dans le cas présent, cf. Annexe II). Les plus petits calibres utilisés auraient pu ainsi favoriser la prise d'alimentation des alevins grâce à une plus grande densité de particules présentes - ou un moindre éloignement particule/alevins (WANKOWSKI, 1981). Ceci permettrait d'expliquer le retard de croissance acusé par le lot nourri au " $\mathrm{J}$ " / 800-1000 lors du premier essai, alors que la structure de population ne semble pas montrer que ce calibre soit trop gros puisque les particules de cette taille sont ingérées ultérieurement par des alevins dont la taille n'a pas varié, ceci probablement suite à un certain "apprentissage".

3. Sur l'ensemble des expériences on observe parfois des différences importantes de performances pour un calibre donné en fonction des aliments - l'aliment " $R$ " semblant permettre les meilleures croissances - cependant dans le cadre d'une telle expérience et en fonction des seules analyses proximales (cf. Annexe l) il n'est pas possible de tirer des conclusions précises d'ordre nutritionnel.

4. L'influence de la taille de la particule alimentaire dépend de l'aliment. Ainsi pour les trois essais, l'aliment " $J$ " permet une croissance meilleure lors du "démarrage" avec les petits calibres $(200-400 \mu$ ), alors que l'on obtient un résultat inverse avec les deux autres aliments. Ceci est difficilement explicable car, d'une part ces résultats ne sont obtenus qu'avec des alevins dont le poids est inférieur à $250 \mathrm{mg}$, et, d'autre part, les différents calibres utilisés proviennent d'une même fabrication et ne montrent pas de difference de composition (cf. Annexe I). Seuls des facteurs physiques liés au broyage ou à la calibration ultérieure pourraient peut-être intervenir: forme, apparence de la particule...

A partir du moment où les alevins sont "démarrés " (taille supérieure à $250 \mathrm{mg}$, ce qui exclut le 1er essai) il semble que pour tous les aliments utilisés le calibre $630-800 \mu$ (PFR $=0.024$ ) permette les meilleures croissances. Il est cependant regrettable de ne pas avoir pu utiliser des calibres plus gros dans les conditions du troisième essai puisque la première expérience semble indiquer que les particules de $800-1000 \mu$ peuvent être ingérées.

On notera que la relation trouvée entre la taille de l'aliment et la taille de l'alevin pour des alevins de 250 a $350 \mathrm{mg}: \mathrm{PFR}=0.021$ a 0,024 , est identique à celle obtenue par WANKOWSKI pour des alevins de taille supérieure ou égale à $4,2 \mathrm{~cm}(670 \mathrm{mg})$. Ce dernier auteur obtenait cependant une fourchette de valeur (PFR de 0,01 à 0,1 ) beaucoup plus grande pour la période de prise d'aliment. Lors de ces essais, le lot témoin non alimenté grossit en bénéficiant probablement d'un apport de proies vivantes par l'alimentation en eau, et les performances des lots alimentés avec des particules de différentes tailles ont été calculées par différence avec le témoin. Il est en conséquence probable que ces données sont moins fiables que celles obtenues pour les alevins plus gros ou que celles que nous avons pu obtenir pour les alevins de $200-350 \mathrm{mg}$.

En conséquence, il nous semble que l'on peut appliquer la formule de WANKOWSKI dès que les alevins ont pris l'habitude de se nourrir sur aliment artificiel (200 mg). 


\section{IV - CONCLUSIONS PRATIQUES}

Pratiquement on conseillera donc de distribuer, pour le démarrage et jusqu'à environ $200 \mathrm{mg}$ en moyenne, des aliments calibrés $400-630 \mu$ (qui permettent les meilleures performances ou pas différentes des meilleures pour les trois aliments utilisés dans cet essai): par la suite, on peut appliquer la formule proposée par WANKOWSKI pour des alevins plus gros: taille de particules en $\mathrm{mm}=0,024 \times$ taille de poisson. Ceci nous permet d'établir pour le saumon atlantique une correspondance entre la granulométrie des aliments (tamis standard) a distribuer et la taille des alevins (tableau 3).

\begin{tabular}{|c|c|}
\hline $\begin{array}{l}\text { Poids de l'alevin } \\
\text { de Salmo salar } \\
\text { en mg }\end{array}$ & $\begin{array}{c}\text { Calibre de l'aliment } \\
\mu\end{array}$ \\
\hline Démarrage à 200 & $400-630$ \\
\hline $200-400$ & $630-800$ \\
\hline $400-750$ & $800-1000$ \\
\hline $750-1300$ & $1000-1250$ \\
\hline
\end{tabular}

Tableau 3 : Calibre d'aliment à distribuer pour des alevins de Salmo salar.

\section{BIBLIOGRAPHIE}

CANYURT, M.A., 1976. Étude de la production et de la qualité de jeunes saumons atlantiques Salmo salar de repeuplement élevés dans différents milieux. Thèse de 3ème cycle Biol. Animale, Université Paul Sabatier, Toulouse, $126 \mathrm{p}$.

FENDERSON, O. and M.R. CARPENTER, 1971. Effects of crowding on the behaviour of juvenile hatchery and wild land-locked atlantic salmon Salmo salar. Anim. Behav., $19,439-447$.

GAIGNON, J.L. et P. PROUZET, 1981. Étude de la production de juvéniles de saumon atlantique. II - Influence de la densité sur l'alevinage. Rapport interne CNEXO/COB, 35 p.

GAIGNON, J.L. et P. PROUZET, 1982 a. Élevage du saumon atlantique (Salmo salar)en écloserie: essai d'utilisation de substrats durant la résorption vitelline. Bull. Fr. Pisc., 287, 1-5.

GAIGNON, J.L. et P. PROUZET, 1982 b. Programme de repeuplement "Sea Ranching " de salmonidés - Production de juvéniles sạison 80-81. III - Production de juvéniles de saumon atlantique: résultats expérimentaux. Rapport scientifique et technique. Département BAP CNEXO/COB.

REFSTIE, T. and A. KITTELSEN, 1976. Effect of density on growth and survival of artificially reared atlantic salmon. Aquaculture, 8, 319-326.

WANKOWSKI, J.W.J., 1979. Morphological limitations, prey size selectivity, and growth response of juvenile atlantic salmon Salmo salar. J. Fish. Biol. 14, 89-100.

WANKOWSKI, J.W.J., 1981. Behavioural aspects of predation by juvenile atlantic salmon Salmo salar on particulate, drifting prey. Anim. Behav., 29, 557-571.

WANKOWSKI, J.W.J. and J.E. THORPE, 1979. The role of food particle size in the growth of juvenile atlantic salmon Salmo salar. J. Fish. Biol. 14, 351-370. 


\section{Annexe I}

Composition des différents aliments utilisés

\begin{tabular}{|c|c|c|c|c|c|c|c|}
\hline Alinisnt & \multicolumn{3}{|c|}{ R } & \multicolumn{4}{|c|}{$F$} \\
\hline $\begin{array}{l}\text { Calibre on } " 1 \\
\text { (ternis standard) }\end{array}$ & $200-400$ & $4001-630$ & $630-800$ & $<200$ & $200-400$ & 400.630 & $000 \cdot 6$ \\
\hline Hernidite on: & 7,0 & 6,3 & 0,7 & 11,01 & 11,02 & 11,01 & $11,0 ?$ \\
\hline Protéings $(\mathrm{N} \times 25,25)$ & 60,2 & 59,1 & 60.0 & 52.09 & 51.05 & 52,00 & 52,015 \\
\hline Lipides & 13,9 & 11.6 & 15.2 & 16,7 & 16,1 & 16.2 & $15, \cong$ \\
\hline Cendres & 19,9 & 12,6 & 13,1 & 8,0 & 7,7 & 7,9 & 7,9 \\
\hline dont Ca & 2.34 & 2,58 & 3,11 & 1,17 & 1,17 & 1,19 & $1, i 7$ \\
\hline $\mathbf{P}$ & 1.68 & 1.68 & 1.83 & 1.01 & 1.01 & 1,09 & 1.01 \\
\hline
\end{tabular}

\begin{tabular}{|c|c|c|c|c|}
\hline Aliment & \multicolumn{4}{|c|}{$-\mathbf{J}$} \\
\hline $\begin{array}{l}\text { Calibre en } \mu \\
\text { (tamis standard) }\end{array}$ & $\begin{array}{l}200 \\
(1)\end{array}$ & $\begin{array}{c}200-400 \\
(1)\end{array}$ & $\begin{array}{c}630-800 \\
(1)\end{array}$ & $\begin{array}{c}1250-1600 \\
(1)\end{array}$ \\
\hline Humidité en \% & 5,04 & 4.08 & 5,04 & 6,02 \\
\hline Protéines $(N \times 6,25)$ & 58 & 56 & 55 & 58 \\
\hline Lipides & 12,01 & 11,00 & 12,03 & 9,09 \\
\hline Cendres & 12,04 & 12,07 & 12,08 & 12,03 \\
\hline dont $\mathrm{Ca}$ & 2,86 & $3, \mathrm{CO}$ & 2,76 & 2,58 \\
\hline $\mathbf{P}$ & 2,02 & 1,96 & 1,88 & 1,76 \\
\hline
\end{tabular}

(1) : Granulométrie commerciale. Le calibre mentionné correspond ì la: classe la plus fréquente de chaque calibre du commerce. 


\section{Annexe II}

Granulométrie des aliments "commerciaux" utilisés (miettes "alevinage" uniquement)

(\% par catégorio)

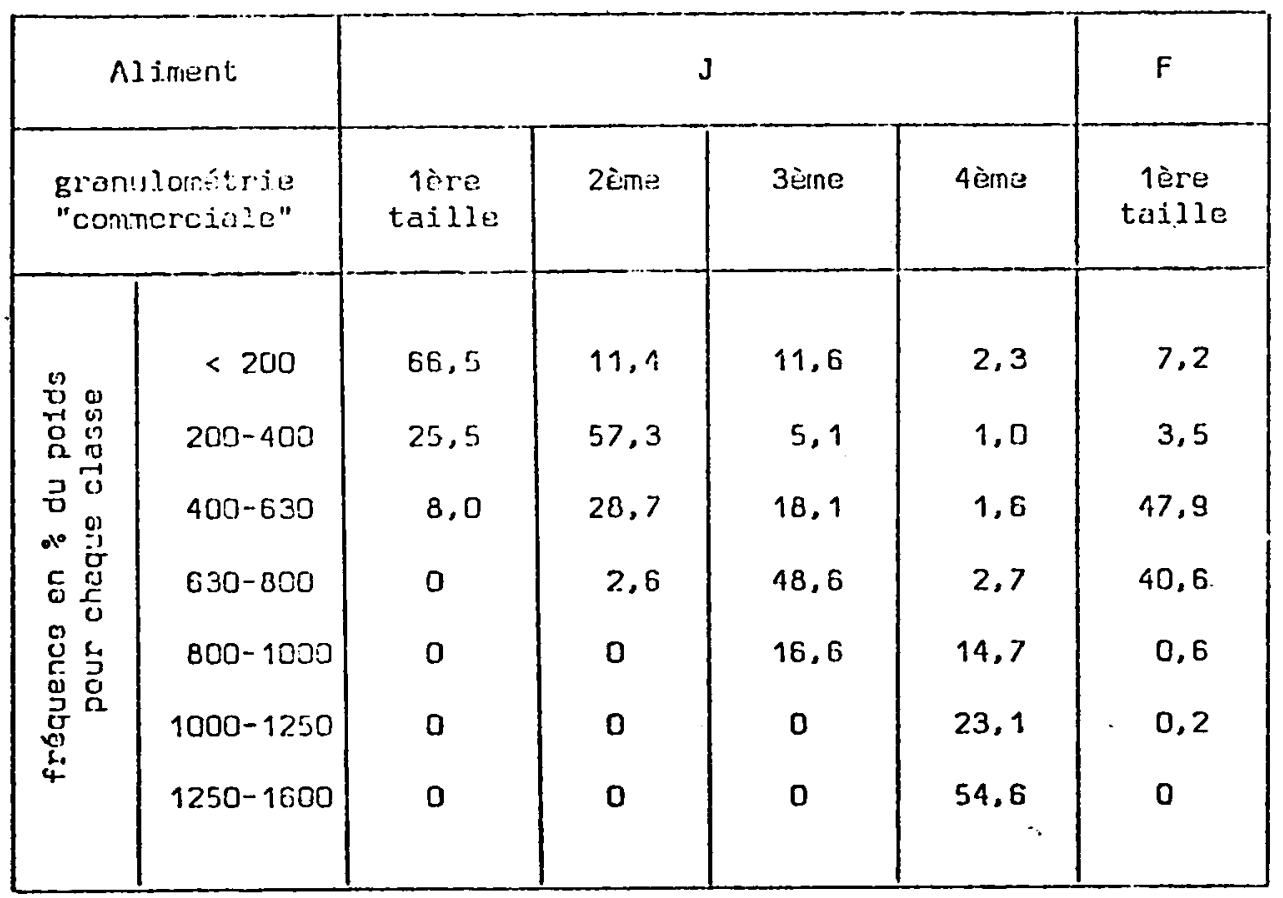

On note la non standardisation des références commerciales en ce qui concerne la granulométrie et on suggèrera donc pour l'avenir qu' un effort soit entrepris dans ce sens.

Sur l'aliment " J ", le poids moyen des miettes de chaque classe de taille est lo suivant :

\begin{tabular}{|c|c|c|c|c|c|c|}
\hline Calibre en $\mu$ & $200-400$ & $400-630$ & $630-800$ & $800-1000$ & $1000-1250$ & $1250-1600$ \\
Poids moyen en $\mathrm{mg}$ & 2,6 & 15,0 & 29,9 & 70,0 & 103,6 & 152,2 \\
\hline
\end{tabular}

